\title{
Modified Finite-Difference Formula for Semivectorial H-Field Solutions of Optical Waveguides
}

\author{
Junji Yamauchi, Member, IEEE, Gen Takahashi, and Hisamatsu Nakano, Fellow, IEEE
}

\begin{abstract}
The propagation constants of rib waveguides are analyzed by the imaginary distance beam-propagation method based on a newly derived finite-difference formula for semivectorial H-fields. The formula ensures a truncation error of $O(\Delta x)^{2}$, provided that the discontinuity lies midway between two mesh points. Comparison with previously published results for a rib waveguide as a classical benchmark shows the effectiveness of the present method.
\end{abstract}

Index Terms-Finite-difference methods, optical beam propagation, optical waveguides.

\section{INTRODUCTION}

$\mathbf{T}$ O IMPROVE accuracy of a propagating beam analysis in a step-index optical waveguide, care has to be exercised in evaluation of the second derivatives of transverse fields, since the accuracy of the conventional finite-difference formula deteriorates at an interface between different refractive indices [1], [2]. In the analysis of a step-index waveguide, a semivectorial approach is often sufficient, unless one needs minor field components [3]. Recently, we have derived an improved formula for semivectorial E-fields [4], which ensures a truncation error of $O(\Delta x)^{2}$.

In this letter, we present a novel finite-difference formula for semivectorial H-fields. To demonstrate superiority of a newly derived formula, we evaluate the normalized propagation constant of rib waveguides [3], using the imaginary-distance beam-propagation method (BPM) [5]-[7]. The results in quasiTE and quasi-TM modes are compared with those obtained by the modal transverse resonance method (MTRM) [3], [8].

\section{FORMULATION}

Three consecutive mesh points shown in Fig. 1 are considered. The interface is at distance $\xi \Delta x$ (with $0<\xi<1$ ) from point $i$. Starting from the one-dimensional Helmholtz equation, we first express the fields $\phi_{i-1}$ and $\phi_{i+1}$ using Taylor-series expansions, as described in [2] and [4]. Let $\phi_{R}$ and $\phi_{L}$ refer to the fields at the infinitesimally right and left of the interface, respectively. The continuity relations at the interface, which

Manuscript received December 31, 1997; revised April 14, 1998.

The authors are with the College of Engineering, Hosei University, Koganei, Tokyo 184, Japan.

Publisher Item Identifier S 1041-1135(98)05384-1.

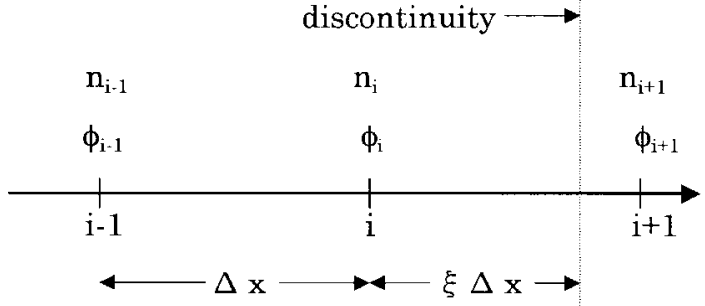

Fig. 1. Mesh points near a discontinuity.

are different from those in E-fields [4], require that

$$
\begin{aligned}
\phi_{R} & =\phi_{L} \\
\frac{\partial \phi_{R}}{\partial x} & =\theta \frac{\partial \phi_{L}}{\partial x} \\
\frac{\partial^{2} \phi_{R}}{\partial x^{2}} & =\frac{\partial^{2} \phi_{L}}{\partial x^{2}}+k^{2}\left(n_{i}^{2}-n_{i+1}^{2}\right) \phi_{L} \\
\frac{\partial^{3} \phi_{R}}{\partial x^{3}} & =\theta\left[\frac{\partial^{3} \phi_{L}}{\partial x^{3}}+k^{2}\left(n_{i}^{2}-n_{i+1}^{2}\right) \frac{\partial \phi_{L}}{\partial x}\right]
\end{aligned}
$$

where $k$ is the free-space wavenumber and $\theta=n_{i+1}^{2} / n_{i}^{2}$. After some manipulations similar to those in [4], we finally obtain

$$
\frac{\partial^{2} \phi_{i}}{\partial x^{2}}=\frac{a \phi_{i-1}+b \phi_{i}+c \phi_{i+1}}{d \Delta x^{2}}+e \frac{\Delta x}{3} \frac{\partial^{3} \phi_{i}}{\partial x^{3}}+O\left(\Delta x^{2}\right)
$$

where

$$
\begin{aligned}
& a=1+(\theta-1)(1-\xi)+m \Delta x^{2} \Gamma \\
& b=-2-(\theta-1)(1-\xi)-m \Delta x^{2}(1+\Gamma) \\
& c=1 \\
& d=1+\frac{1}{2}(\theta-1)(1-\xi)(2 \xi+1)+\frac{1}{2} m \Delta x^{2} \Gamma \\
& e=-(\theta-1) \xi(1-\xi)(2 \xi-1) / d
\end{aligned}
$$

in which

$$
\begin{aligned}
m & =\frac{1}{2} k^{2}(1-\xi)^{2}\left(n_{i}^{2}-n_{i+1}^{2}\right) \\
\Gamma & =\xi+\theta \frac{1-\xi}{3} .
\end{aligned}
$$

It is worth mentioning that the coefficient of $\partial^{3} \phi_{i} / \partial x^{3}$ vanishes when either $\xi=0.5$ or 0 (note that $\xi=0$ is approximately realized in the limit where $\xi$ approaches zero). Equation (1) reduces to Stern's formula [1] when we neglect the $\partial^{3} \phi_{i} / \partial x^{3}$ term making $m=0$ and $\xi=0.5$. A formula 


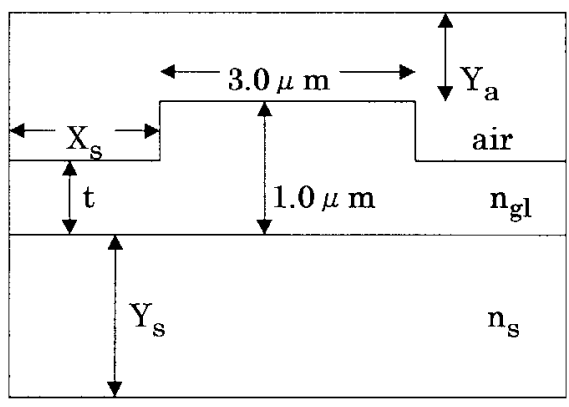

Fig. 2. Rib waveguide geometry.

similar to (1) is also derived when the discontinuity lies between $i-1$ and $i$.

To assess the accuracy of the newly derived formula, we evaluate the eigenmode of a step-index waveguide. The fundamental mode is obtained by the imaginary distance BPM in which the coordinate $z$ in the propagation direction is changed to $\mathrm{j} \tau$. The alternating-direction implicit method is employed for the analysis of a three-dimensional structure.

The effective index can be evaluated by the growth in the field amplitude [6], i.e.,

$$
\begin{aligned}
& \beta(\tau)=n_{0} k \\
& +\frac{\ln \left\{\int \phi(x, y, \tau+\Delta \tau) d x d y\right\}-\ln \left\{\int \phi(x, y, \tau) d x d y\right\}}{\Delta \tau} .
\end{aligned}
$$

For evaluation of the effective index, the reference index $n_{0}$ has to be a value close to the exact one. It should be noted, however, that the exact value is unknown in practice. In this letter, we adopt a new technique of iteratively renewing $n_{0}$ [9]. This technique leads to the advantage that the effective index converges automatically.

\section{RESULTS}

We consider a rib waveguide shown in Fig. 2. This configuration has been used as a classical benchmark [3]. The configuration parameters are $n_{s}=3.40, n_{g l}=3.44$, rib width $=3.0 \mu \mathrm{m}$, central rib height $=1.0 \mu \mathrm{m}$, and lateral height $=t$ varying from $0.1-0.9 \mu \mathrm{m}$. The computational domain parameters are $Y_{a}=0.5 \mu \mathrm{m}$ above the top of rib, $Y_{s}=$ $3.0 \mu \mathrm{m}$ below the guiding layer, $X_{s}=2.5 \mu \mathrm{m}$ (or $5.5 \mu \mathrm{m}$ for $t=0.9 \mu \mathrm{m}$ ) aside from the rib lateral side. A wavelength of $\lambda=1.15 \mu \mathrm{m}$ is used. A transparent boundary condition often used in a propagating beam analysis is not necessarily needed for this analysis, since the fields other than the fundamental mode decay rapidly as they propagate along the imaginary axis. Hence, no special boundary condition is imposed at the edge of the computational window, i.e., zero boundary terms are used instead of a transparent boundary condition.

The effective index is defined by $n_{\mathrm{eff}}=\beta / k$ and the normalized propagation constant is $B=\left(n_{\text {eff }}^{2}-n_{s}^{2}\right) /\left(n_{g l}^{2}-n_{s}^{2}\right)$. Fig. 3 shows the convergence behavior of $B$ for the quasi-TE and quasi-TM modes as a function of transverse mesh size $\Delta(=\Delta x=\Delta y)$. In this analysis $t$ is chosen to be $0.5 \mu \mathrm{m}$.

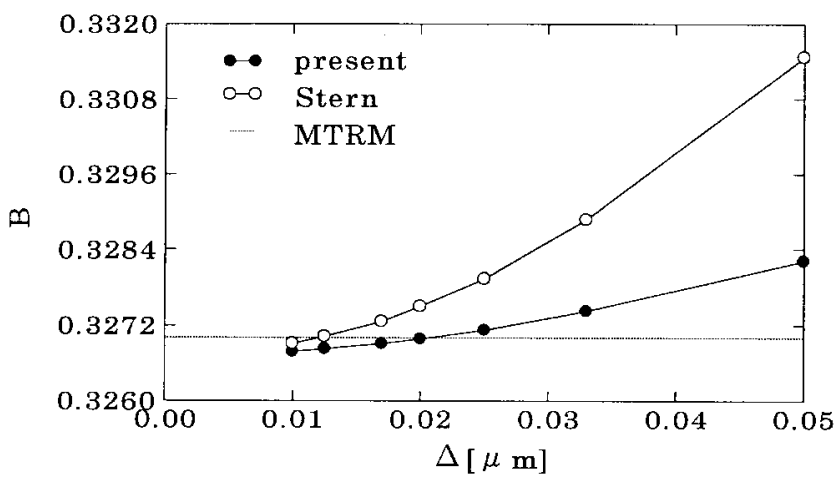

(a)

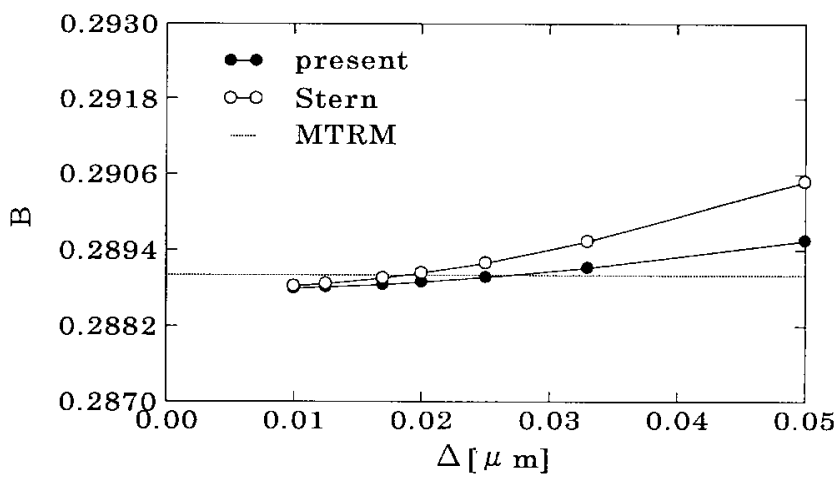

(b)

Fig. 3. Convergence behavior of normalized propagation constant $B$ as a function of transverse mesh size $\Delta$.(a) Quasi-TE mode. (b) Quasi-TM mode.

For comparison, the results obtained using Stern's formula are also shown. It is found that the present formula achieves faster convergence.

Table I tabulates the $B$ values obtained from the present technique in both the quasi-TE and quasi-TM modes. The transverse mesh size is fixed to be $\Delta=0.02 \mu \mathrm{m}$. The values derived with the modal transverse resonance method $(\mathrm{MTRM})$ and the deviations $\left(B-B_{\mathrm{MTRM}}\right) \times 10^{4}$ are also presented. The $B_{\text {MTRM's have been independently computed }}$ by Sudbø and Vassallo, and the four-digit values are believed to be exact [3], [8] (note that the MTRM is a full-vectorial eigenmode solver). It is found that the present results agree well with those obtained from the MTRM. Further comparison with other data summarized in [3, Table I] demonstrates the effectiveness of the present method. The maximum deviation, which is observed in the quasi-TE mode, is +3 for $t=0.7$ and $0.9 \mu \mathrm{m}$. Incidentally, we have also calculated the $B$ values using the E-field formulation [4]. The results agree with those obtained from the $\mathrm{H}$-field formulation up to five-digit values.

It should be noted that the $B$ values for $\Delta=0.02 \mu \mathrm{m}$ do not converge completely. Since the error is almost proportional to $\Delta^{2}$, we can estimate more exact $B$ 's at $\Delta=0$ by an extrapolation technique [3], [10]. Table I also presents the extrapolated value $B_{\mathrm{EXTR}}$. In general, the $B_{\mathrm{EXTR}}$ 's are slightly less than the $B_{\mathrm{MTRM}}$ 's. This is considered to be due to negligence of coupling terms in a semivectorial analysis. The fact that the semivectorial analysis tends to yield an effective index slightly less than that in the full-vectorial analysis is also found in [11]. 
TABLE I

Normalized Propagation CONSTANTS ANd DeVIaTions From MTRM

\begin{tabular}{c|ccrcc} 
& \multicolumn{6}{|c}{ quasi-TE mode } \\
$\mathrm{t}$ & MTRM & \multicolumn{1}{c}{$\mathrm{B}$} & deviations & B ExtR & deviations \\
\hline 0.1 & 0.3019 & 0.3020 & +1 & 0.3018 & -1 \\
0.3 & 0.3110 & 0.3111 & +1 & 0.3108 & -2 \\
0.5 & 0.3270 & 0.3270 & 0 & 0.3267 & -3 \\
0.7 & 0.3510 & 0.3513 & +3 & 0.3510 & 0 \\
0.9 & 0.3883 & 0.3886 & +3 & 0.3884 & +1
\end{tabular}

\begin{tabular}{c|ccccc} 
& \multicolumn{5}{|c}{ quasi-TM mode } \\
$\mathrm{t}$ & MTRM & B & deviations & BExTR & deviations \\
\hline 0.1 & 0.2674 & 0.2676 & +2 & 0.2674 & 0 \\
0.3 & 0.2751 & 0.2751 & 0 & 0.2750 & -1 \\
0.5 & 0.2890 & 0.2889 & -1 & 0.2888 & -2 \\
0.7 & 0.3107 & 0.3106 & -1 & 0.3105 & -2 \\
0.9 & 0.3455 & 0.3455 & 0 & 0.3454 & -1 \\
\end{tabular}

\section{CONCLUSION}

We have derived an improved finite-difference formula for semivectorial $\mathrm{H}$-fields in step-index optical waveguides. The derived formula ensures a truncation error of $O(\Delta x)^{2}$, provided that the discontinuity lies midway between two mesh points. The propagation constants of rib waveguides for a classical benchmark test are evaluated using the imaginary distance BPM in both the quasi-TE and quasi-TM modes. The obtained results are compared with the values derived with the modal transverse resonance method (MTRM). The present formula can be used for a propagating beam analysis, while maintaining the accuracy comparable to that of the MTRM.

\section{REFERENCES}

[1] M. S. Stern, "Semivectorial polarized $H$ field solutions for dielectric waveguides with arbitrary index profiles," Proc. Inst. Elect. Eng., pt. J, vol. 135 , no. 5 , pp. $333-338,1988$.

[2] C. Vassallo, "Improvement of finite difference methods for step-index optical waveguides," Proc. Inst. Elect. Eng., pt. J2, vol. 139, no. 2, pp. 137-142, 199.

[3] __ "1993-1995 optical mode solvers," Optical and Quantum Electron., vol. 29, no. 2, pp. 95-114, 1997.

[4] J. Yamauchi, M. Sekiguchi, O. Uchiyama, J. Shibayama, and H. Nakano, "Modified finite-difference formula for the analysis of semivectorial modes in step-index optical waveguides," IEEE Photon. Technol. Lett., vol. 9, pp. 961-963, July 1997.

[5] D. Yevick and B. Hermansson, "New formulations of the matrix beam propagation method: Application to rib waveguides," IEEE J. Quantum Electron., vol. 25, no. 2, pp. 221-229, 1989.

[6] C. L. Xu, W. P. Huang, and S. K. Chaudhuri, "Efficient and accurate vector mode calculations by beam propagation method," J. Lightwave Technol., vol. 11, no. 7, pp. 1209-1215, 1993.

[7] S. Jüngling and J. C. Chen, "A study and optimization of eigenmode calculations using the imaginary-distance beam-propagation method," IEEE J. Quantum Electron., vol. 30, pp. 2098-2105, Sept. 1994.

[8] A. S. Sudb $\varnothing$, "Film mode matching: A versatile numerical method for vector mode field calculations in dielectric waveguides," J. Eur. Opt. Soc. A, Pure Appl. Opt., vol. 2, pp. 211-233, 1993.

[9] J. Shibayama, M. Sekiguchi, J. Yamauchi, and H. Nakano, "Eigenmode analysis of optical waveguides by an improved finite-difference imaginary-distance beam propagation method," IEICE Trans., vol. J81C-I, no. 1, pp. 9-16, 1998.

[10] W. W. Lui, W. P. Huang, and K. Yokoyama, "Precision enhancement for optical waveguide analysis," J. Lightwave Technol., vol. 15, pp. 391-396, Feb. 1997.

[11] P. Lüsse, K. Ramm, and H.-G. Unger, "Comparison of a vectorial and new semivectorial finite-difference approach for optical waveguides," Optical and Quantum Electron., vol. 29, no. 2, pp. 115-120, 1997. 